\title{
Tia Leading to the Diagnosis Amelanotic Metastatic Gastric Melanoma
}

\author{
Shaheer Siddiqui ${ }^{1}$, Tarun Jain ${ }^{2}$, Zhenjian Cai ${ }^{3}$ and Scott Larson ${ }^{4 *}$ \\ ${ }^{1}$ Department of Gastroenterology \& Hepatology, University of Texas, McGovern School of Medicine, USA \\ ${ }^{2}$ Department of Resident Internal Medicine, University of Texas, McGovern School of Medicine, USA \\ ${ }^{3}$ Department of Assistant Professor Pathology, University of Texas, McGovern School of Medicine, USA \\ ${ }^{4}$ Department of Assistant Professor Gastroenterology \&Hepatology, University of Texas, McGovern School of Medicine, USA
}

*Corresponding author: Scott Larson, Department of Assistant Professor Gastroenterology \&Hepatology, LBJ General Hospital, University of Texas, McGovern School of Medicine, USA.

Received Date: August 07, 2019

Published Date: August 13, 2019

\begin{abstract}
Melanoma metastatic to the GI tract is a rare disease. Due to hematogenous spread it usually presents as multiple pigmented lesions in the GI tract. We present a case of a 62 years old gentleman diagnosed with melanoma presenting to the hospital with GI bleed and severe anemia. Endoscopic evaluation revealed a large gastric polypoid mass which proved to be a metastatic melanoma diagnosed 39 months after the initial diagnosis of primary melanoma. The rare presentation of our case is the amelanotic nature of the metastatic melanoma appearing as a gastric mass.
\end{abstract}

\section{Introduction}

The GI tract is one of the most common sites of metastasis of malignant melanoma and can present as an initial diagnosis or discovered years after the initial diagnosis [1]. Only $4 \%$ of the patient population with malignant melanoma will have GI involvement during their lives otherwise $60 \%$ of metastatic melanomas to the GI tract are discovered postmortem [2]. Amongst all the metastatic sites of the melanoma secondary gastric lesions are only reported to be between $0.2 \%$ to $1.7 \%[3,4,5]$.

\section{Case Report}

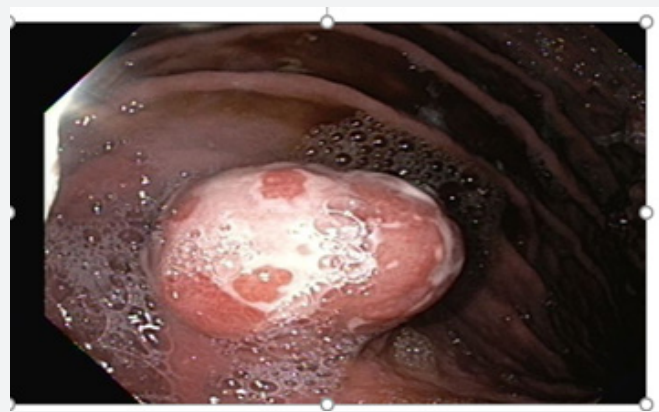

Figure 1A: Endoscopic appearance of the polypoid gastric mass in the body of the stomach with friable, necrotic and ulcerated mucosa.

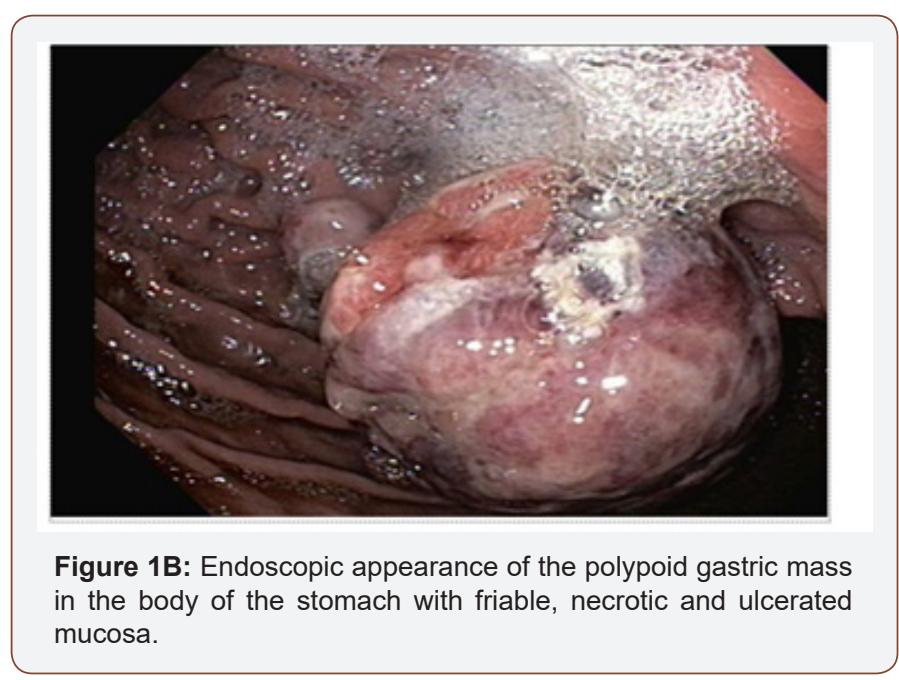

We are presenting a case of a 62 years old gentleman who was initially diagnosed with metastatic melanoma stage IIIb in 4/2015 and underwent wide local excision. Biopsy results revealed negative margins. No nodal metastasis was noted. Because of localized disease the patient underwent radiation therapy that was completed in 7/2015. Patient had a close follow up with oncology and after undergoing comprehensive physical examination, laboratory studies and imaging it was revealed that patient had 
no evidence of disease. In January of 2018 patient developed a subcutaneous nodule in the left flank area which was biopsied but was negative for malignancy. Patient underwent a CT scan of chest, abdomen and pelvis and PET scan in 2/2018 to look for metastatic disease but did not reveal any significant findings. In $4 / 2018$ patient suffered a stroke and was started on dual antiplatelet therapy. After few months the patient started feeling extremely week and fatigued complaining of having black tarry stools. In 7/2018 patient visited his PCP for evaluation and was found to have severe anemia with a hemoglobin of $4.5 \mathrm{~g} / \mathrm{dL}$. Patient was referred to the emergency room for further evaluation. Patient was admitted under the service of Internal Medicine and underwent blood transfusions. Patients anti platelet therapy was put on hold. Gastroenterology was consulted as there was a high suspicion of an upper GI bleed. An EGD revealed a large $6 \mathrm{~cm}$ polypoid gastric mass with one satellite lesion with extremely friable, necrotic and ulcerated mucosa (Figure $1 \mathrm{~A}$ and 1B). Gastric mass was not removed because of the large size and also increased risk of bleeding. A hot snare was used for tissue acquisition which was sent to pathology.

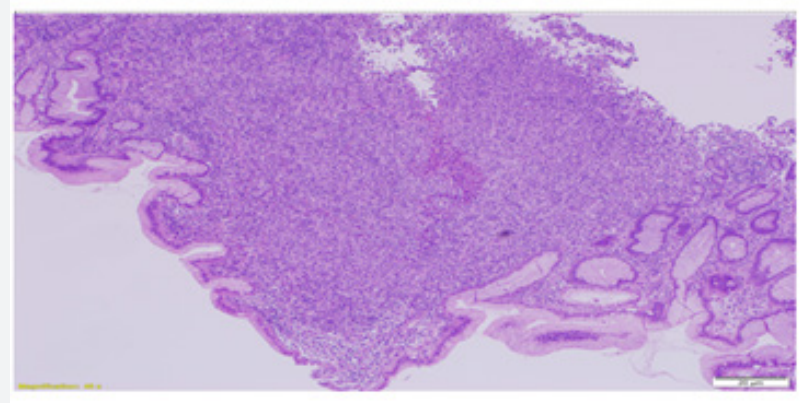

Figure 2: Melanoma cells invading the gastric mucosa.

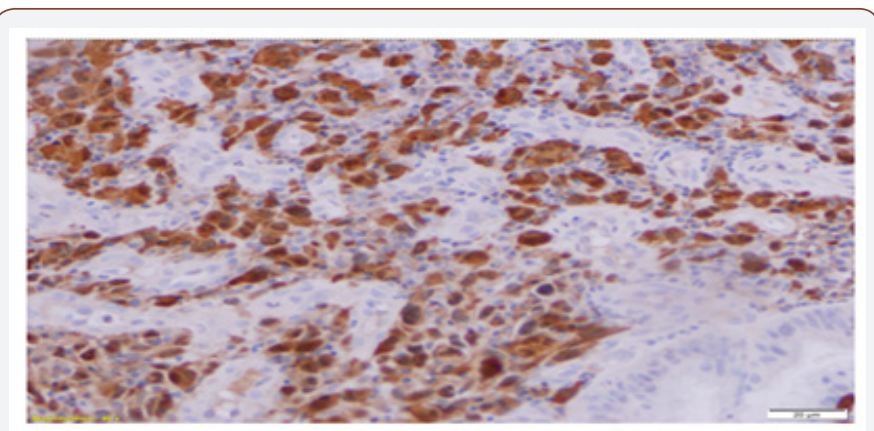

Figure 3: Cancer cells positive for S100

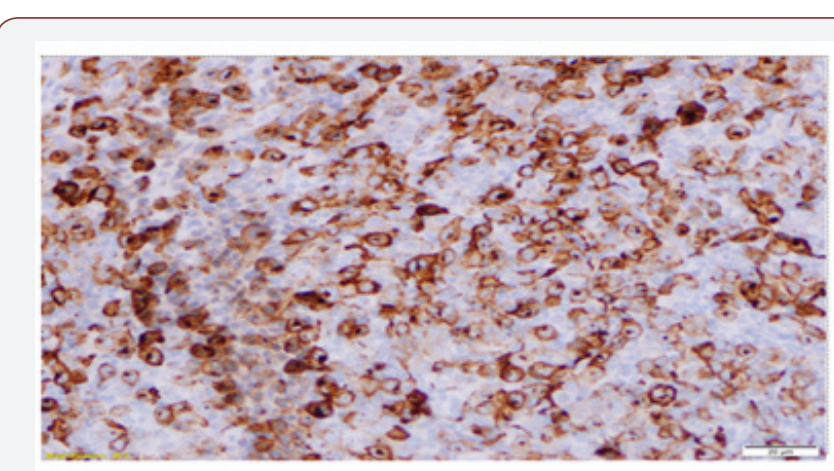

Figure 4: Cancer cells positive for HMB 45. 
and anemia of unknown etiology should raise concerns about metastatic melanoma to the GI tract. Prompt evaluation, endoscopic and histological diagnosis is extremely important in formulating a treatment plan for better outcomes as malignant metastatic melanomas represents a poor overall prognosis.

\section{Conclusion}

Our case presents a very rapid progression of the melanoma from apparently no disease to finding of metastatic melanoma presenting as upper GI bleed with discovery of large polypoid gastric mass within six months. The most interesting aspect of our case is amelanotic nature of the gastric mass. On initial endoscopic evaluation it appeared as a gastric polyp and it was the biopsy that proved the gastric mass as malignant melanoma. We might not have even discovered the gastric melanoma if the patient did not present with upper GI bleed which was probably due to initiation of dual anti platelet therapy and oozing of blood form the friable mucosa. Hence the diagnosis of amelanotic malignant melanoma presenting as large polypoid gastric mass which in itself is a rare entity.

\section{Acknowledgements}

None.

\section{Conflict of Interest}

No conflict of interest.

\section{References}

1. Köklü S, Gültuna S, Yüksel I, Başar Ö, Üsküdar O, et al. (2008) Diffuse Gastroduodenal Metastasis of Conjunctival Malignant Melanoma. The American Journal of Gastroenterology 103(5): 1321-1323.

2. Patel JK, Didolkar MS, Pickren JW, Moore RH (1978) Metastatic pattern of malignant melanoma: a study of 216 autopsy cases. The American Journal of Surgery 135(6): 807-810.

3. Menuck LS, Amberg JR (1975) Metastatic disease involving the stomach. The American Journal of Digestive Diseases 20(10): 903-913.

4. Ga Hee Kim, Ji Yong Ahn, Hwoon-Yong Jung, Young Soo Park, Min Ju Kim, et al. (2015) Clinical and Endoscopic Features of Metastatic Tumors in the Stomach. Gut and Liver 9(5): 615-622.

5. Zhao HD, Wang G, Wang T, Liang X, Zhou L, et al. (2014) Gastrointestinal tract metastasis from tubulolobular carcinoma of the breast: a case report and review of the literature. OncoTargets and Therapy 7: 435440 .

6. Suganuma T, Fujisaki J, Hirasawa T, Ishiyama A, Yamamoto Y, et al. (2013) Primary amelanotic malignant melanoma of the small intestine diagnosed by esophagogastroduodenoscopy before surgical resection. Clinical Journal of Gastroenterology 6(3): 211-216.

7. Reintgen DS, Thompson W, Garbutt J, Seigler HF (1984) Radiologic, endoscopic, and surgical considerations of melanoma metastatic to the gastrointestinal tract. Plastic and Reconstructive Surgery 75(4): 95(6): 635-639.

8. Gilg MM, Gröchenig HP, Schlemmer A, Eherer A, Högenauer C, et al. (2018) Secondary tumors of the GI tract: origin, histology, and endoscopic findings. Gastrointestinal Endoscopy 88(1): 151-158.

9. Weigt J, Malfertheiner P (2015) Metastatic Disease in the Stomach Gastrointestinal Tumors 2(2): 61-64. 